\title{
Pathophysiology of intravascular coagulation and fibrinolysis
}

\author{
A A SHARP \\ From the Department of Haematology, John Radcliffe Hospital, Oxford
}

When tissue is damaged one reaction is the initiation of the haemostatic mechanism. This involves initially platelet interaction with the damaged vessel walls, platelet aggregation and release at the sites of damage, and subsequent local fibrin formation due to the activation of the coagulation cascade after the 'release' of various factors from the activated platelets -namely, $\mathrm{Ca}^{++}$ions and phospholipid $\left(\mathrm{PF}_{3}\right)$.

In trauma or disease in man the injury to tissue directly and indirectly can invoke a similar mechanism and, if the stimulus is very strong, the normally protective haemostatic mechanism is activated to such an extent that serious destruction of platelets and significant fibrin formation may occur both at the site of injury and also in normal tissue. In order to limit the spread of fibrin the fibrinolytic mechanism is activated along with or as a consequence of fibrin formation. This 'pathological' activation of the physiological haemostatic process has been called intravascular coagulation and fibrinolysis (ICF), a term which is considered by some to be synonymous with the diffuse intravascular coagulation syndrome (DIC). Either term has been used to define the explosive and sometimes fatal activation of the platelet/coagulation sequence as a complication of infections, malignancy, trauma, and immune complex disease in man. ${ }^{1}$ A similar sequence may also complicate abnormal events in pregnancy for example, abruptio placentae and septic abortion. In each instance the platelet, coagulation, fibrinolysis activation is the sequel to tissue damage or the introduction into the blood of a 'trigger'.

Experimentally various triggers have been found to produce ICF/DIC in animals which is almost identical to that seen as a complication of disease in man. ${ }^{2}$ 'Direct' triggers in the form of tissue thromboplastin or snake venoms have been described which activate the coagulation cascade directly. ${ }^{1}$ 'Indirect' triggers, endotoxin and immune complexes, require an interaction with coagulation proteins (XII) or platelets/white cells before activating the platelet/ fibrin interaction. Further these indirect triggers may also first produce tissue or vessel wall damage, invoking a direct activation as well as an indirect effect.

While the ICF/DIC syndrome is considered as a separate entity from conventional forms of arterial and venous thrombosis the pathophysiology of both is similar and may represent only a variable expression of the degree of activation. ${ }^{3}$

Whatever the trigger the sequence of events leads to platelet activation in the vessel wall or in the circulating blood leading to destruction of circulating platelets and eventual thrombocytopenia. If the stimulus is sufficient local or diffuse activation of the coagulation cascade (directly or indirectly) leads to thrombin formation or the conversion of fibrinogen to fibrin. The resultant reaction may be mild and detectable only by laboratory tests or may be so strong that both platelets and coagulation factors are consumed, leading to a tendency to abnormal bleeding (consumption coagulopathy) or to the widespread dissemination of fibrin with significant irreversible tissue damage (DIC) (Fig. 1). An acute stimulus (amniotic fluid embolism) can produce this reaction in minutes while a chronic one (for example, dead fetus syndrome, malignancy) the evolution of change may take some weeks.

\section{Fibrin formation}

The ultimate result of activation of the coagulation cascade is thrombin formation with interaction with platelets, fibrinogen, and Factor XIII (plasma transglutaminase). Fibrin formation is initiated by cleavage of fibrinopeptide A from the A $\alpha$ chains of fibrinogen. Gaffney ${ }^{4}$ and Nossel and Kaplan ${ }^{5}$ have shown that this first cleavage allows the formation of fibrin monomer which can complex with fibrinogen to form soluble fibrin monomer complexes (SFMC) or to lead to fibrin formation (FN) $\mathbf{P}_{1}$ or (fibrin I) (Fig. 2). This fibrin is probably not cross-linked and when attacked by plasmin releases a B $\beta$ 1-42 fragment from the $\beta$ chain which can be detected by immunoassay (Fig. 2). Other products of lysis are found which are similar to those obtained when 


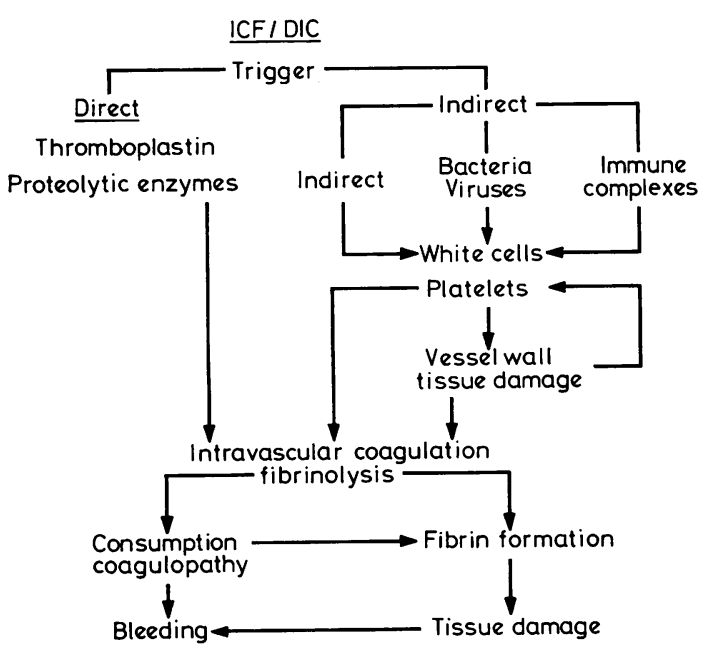

Fig. 1 Sequence of ICF/DIC.

fibrinogen is attacked by plasmin-namely, the fragments $X, Y, D$, and $E$ in their separate forms. A similar situation exists in man when the purified snake venoms Ancrod or Defibrase are used as therapeutic anticoagulants. These agents also act directly on fibrinogen, releasing the A peptide from fibrinogen and so producing a form of 'soluble' fibrin which is rapidly lysed. This reaction results in the complete destruction of fibrinogen. ${ }^{4}$

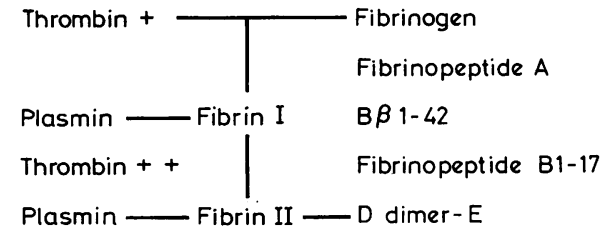

Fig. 2 Thrombin-plasmin, fibrinogen-fibrin interaction (After Nossel and Kaplan ${ }^{5}$.

If the amount of thrombin generated is small or diluted in fast-flowing blood the sequence may end at this stage. But when the concentration of thrombin is increased by explosive activation of the coagulation cascade or owing to stasis or turbulence, the B peptide is split from the $B \beta$ chain. This release is associated with the formation of (FN) $\mathrm{P}_{2}$ or fibrin II and the activation of factor XIII with resultant cross-linking so that fibrin II becomes stabilised and less susceptible to the action of plasmin. When such fibrin is lysed by plasmin the evidence of cross-linking is found by the presence of the complex D dimer-E. 4

This discovery leads to the possibility that in man mild ICF is likely to lead to the formation of fibrin I and the recognisable X, Y, D, E products of plasmin digestion. Further, this sequence of events, if progressive, can lead to a consumption of platelets and fibrinogen without detectable fibrin formation in tissues but with an increased risk of severe bleeding (consumption coagulopathy). This can also occur in continuous chronic ICF-for example, malignancy, dead fetus syndrome.

When the concentration of thrombin is sufficient fibrin II will form and be deposited locally (thrombosis) or in a disseminated form, and this cross-linked stable fibrin is more likely to form thrombi with resultant local or widespread tissue damage-that is, true DIC.

\section{Fibrinolysis}

In the ICF/DIC syndrome the fibrinolytic mechanism activates in both mild and severe examples. Whether this activation occurs at the same time as initiation of the coagulation sequence or is simply the result of intravascular fibrin formation is still conjecture. Whatever the mechanism the production of activator in the blood or from damaged endothelium allows fibrin to be lysed by the collective binding of activator, plasminogen, and plasmin to the fibrin molecule, so protecting the active plasmin from the effect of antiplasmin (Fig. 3). This activation limits the amount of fibrin formed and so in this context is protective.

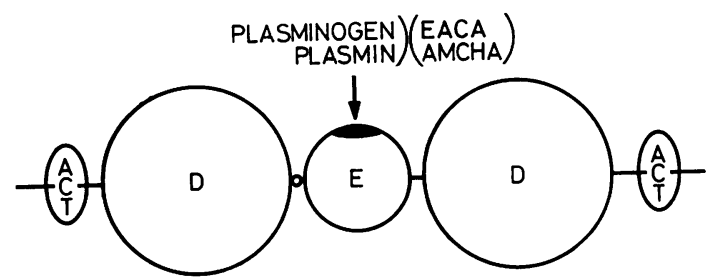

Fig. 3 Fibrinolysis. Binding of activator, plasminogen, and plasmin to fibrinogen molecule. Act $=$ activator. $E A C A=6$-aminocaproic acid. $A M C H A=$ tranexamic acid.

There is no convincing evidence that primary activation of the fibrinolytic mechanism can by itself produce those changes in the blood such as are found in ICF except when therapeutic fibrinolysis is induced by excess activator (urokinase) or the formation of activator by streptokinase.

\section{Clinical aspects of ICF/DIC}

ICF/DIC occurs in a wide variety of diseases. ${ }^{1}$ While the general opinion is that the activation of the 
platelet/coagulation sequence leads to the formation of platelet/fibrin masses locally or diffusely in the body, and so damages tissue, ${ }^{6}$ doubts have often been expressed based on the failure to find fibrin deposition at necropsy.?

This conflict of opinion may be due to the disappearance of fibrin by lysis after it has damaged tissue or to actual post-mortem lysis of fibrin due to the massive release of activator from endothelium after death. Alternatively the clinical expression of DIC might be the consequence of the underlying disease or due to the activation of the other triggered enzyme systems-for example, kinins or complement. Whatever the explanation the clinical expression of $\mathrm{ICF} / \mathrm{DIC}$ is very variable. The common presentation is abnormal bleeding due to depletion of platelets and the coagulation factors. This may be severe and life threatening in obstetrics and trauma or may show more commonly as excessive subcutaneous purpura or bruising. In more severe examples there is evidence of multiple organ damage-for example, skin (gangrene), brain (coma), heart (failure or arrest), lungs (hypoxia), liver (jaundice), kidney (oliguria or anuria), and muscle (acidosis)producing a wide variety of clinical signs (Table). There is no doubt that when such diffuse damage accompanies evidence of ICF in the blood prognosis is much worse. ${ }^{8}$

\section{Clinical features of IFC/DIC}

Heart failure
Cyanosis
Dyspnoea
Oliguria or anuria
Jaundice
Thrombosis
Gangrene
Bleeding
Purpura

If the clinical presentation is analysed in relation to the above hypothesis it would seem that these patients, when the ICF/DIC syndrome is initiated by the direct 'thromboplastin trigger' alone (for instance, in obstetrics and malignancy), are more likely to have ICF alone without evidence of DIC. The changes in the blood-thrombocytopenia, fibrinogen depletion, a low level of fibrinogen, and fibrin I split products-suggest that in these syndromes only fibrin $I$ is formed. When the indirect triggers act via their mediators (as in endotoxaemia and immune complex disease) or by producing tissue damage or massive platelet activation DIC is more likely to evolve as a consequence of the formation of fibrin II, and with consequent tissue damage.

\section{Treatment of ICF/DIC}

The results of various forms of treatment have tended only to confuse our understanding of the exact sequence of events in the pathophysiology of this syndrome. Obviously treating the underlying disease which has provoked the release of trigger is imperative and often successful provided the consequent tissue damage is not too extensive. When ICF/DIC evolves as a complication of disease there may be significant blood loss into damaged tissue and also increased permeability of damaged vessels may lead to plasma transudation and a significant reduction of blood volume, haemoconcentration, and consequent poor oxygen transport to already damaged tissue. Therefore it is of prime importance to restore the effective circulating blood volume as quickly as possible.

If thombin production is as crucial as all evidence suggests then anticoagulant therapy (for example, with heparin) should be the treatment of choice. Yet after many studies there is no clear evidence that heparin has been of any benefit. It may actually provoke further bleeding. ${ }^{7}$ But this may be because too often DIC is diagnosed only after tissue damage has occurred and such therapy would be unlikely to have any effect on formed fibrin or damaged tissue. If ICF/DIC could be predicted, heparin used prophylactically might be successful-for example, in cases of septicaemia, incompatible blood transfusion, or amniotic fluid embolism. Heparin has been shown to reverse the coagulation changes in some cases of chronic ICF/DIC associated with malignancy. ${ }^{9}$ Replacement therapy with fresh frozen plasma or concentrates is indicated when bleeding is the sequel of severe depletion of coagulation factors. There is no convincing evidence that such replacement can promote further fibrin formation and tissue damage.

Within the context of this symposium the role of specific inhibitors of the fibrinolytic mechanism must be considered, but if the theories of pathophysiology proposed above are correct their use is contraindicated.10 Inhibition of fibrinolysis might prevent the lysis of fibrin and so encourage tissue damage. Nevertheless, there are a few instances where their use may be justified-for example, when ICF is associated with an excessive fibrinolytic response and when replacement therapy fails to stop severe bleeding in, say, liver disease and carcinoma of the prostate with metastases.

It is tempting to speculate that if the hypothesis relating to fibrin I and II defined by Gaffney ${ }^{4}$ and Nossel and Kaplan ${ }^{5}$ is tenable then fibrinolytic inhibitors may deter the consumption of fibrinogen or fibrin I by secondary lysis and prevent or reduce 
the serious and sometimes catastrophic bleeding that may result. This problem will be answered only by a properly designed therapeutic trial, which would be difficult to design in relation to this widely variable syndrome.

\section{Conclusion}

The problem of intravascular coagulation and fibrinolysis continues to fascinate and puzzle. While more is being found out about its pathophysiology we cannot progress until we determine the exact pathways of activation, coagulation, and thrombin production in this condition and whether in fact it is fibrin deposition that causes the associated extensive organ malfunction.

\section{References}

${ }^{1}$ Sharp AA. Diagnosis and management of disseminated intravascular coagulation. $\mathrm{Br}$ Med Bull 1977;33: 265-72.

${ }^{2}$ Evensen SA, Hjort PF. Pathogenesis of disseminated intravascular coagulation. In Hematology, 1970. Plenary Sessions and Scientific Contributions of the XIII International Congress of Hematology, Munich, 1970. Munich: JF Lehmann, 1970:109-20.
${ }^{3}$ Stewart GJ. Role of hypercoagulability in thrombosis. Br J Haematol 1978;40:359-62.

${ }^{4}$ Gaffney PJ. Fibrin-plasmin intereactions. A synthesis of molecular and clinical observations. In: Gaffney PJ, Balkuv-Ulutin S, eds. Fibrinolysis: current fundamental and clinical concepts. London: Academic Press, 1978:109-28.

${ }^{5}$ Nossel HL, Kaplan KL. Simultaneous measurement of thrombin and plasmin proteolysis of fibrinogen and of platelet release. In: Bing DH, ed. The chemistry and physiology of human plasma proteins. Oxford: Pergamon Press, 1979:97-110.

${ }^{6}$ Colman RW, Robboy SJ, Minna JD. Disseminated intravascular coagulation and reappraisal. Ann Rev Med 1979;30:359-74.

${ }^{7}$ Mant MJ, King EG. Severe acute disseminated intravascular coagulation. Am J Med 1979;67:557-63.

${ }^{8}$ Siegal T, Seligsohn U, Aghai E, Modan M. Clinical and laboratory aspects of disseminated intravascular coagulation (DIC): a study of 118 cases. Thrombos Haemostas 1978;39:122-34.

${ }^{9}$ Merskey, C. Defibrination syndrome. In: Biggs R, ed. Human blood coagulation. Haemostasis and thrombosis. Oxford: Blackwell Scientific Publications, 1972:444-75.

${ }^{10}$ Chesterman CN. Fibrinolysis and disseminated intravascular coagulation. In: Gaffney PJ, Balkuv-Ulutin S, eds. Fibrinolysis: current fundamental and clinical concepts. London: Academic Press, 1978:157-72. 